\title{
Fuzzy Model-Based Asynchronous Control for Markov Switching Systems with Stochastic Fading Channels
}

\author{
Fayuan Wu, ${ }^{1}$ Jinhui Tang, ${ }^{1}$ Zhuang Liu, ${ }^{1}$ Qi Xiao, ${ }^{2}$ Xiaodong Zheng, ${ }^{3}$ and Shuangsi Xue $\mathbb{i D}^{3}$ \\ ${ }^{1}$ Electric Power Research Institute of State Grid Jiangxi Electric Power Co.Ltd., Nanchang 330096, China \\ ${ }^{2}$ State Grid Jiangxi Electric Power Co.Ltd., Nanchan 330096, China \\ ${ }^{3}$ School of Electrical Engineering, Xi'an Jiaotong University, Xi'an 710049, China \\ Correspondence should be addressed to Shuangsi Xue; xssxjtu@stu.xjtu.edu.cn
}

Received 6 August 2020; Revised 24 August 2020; Accepted 8 September 2020; Published 18 September 2020

Academic Editor: Shuping He

Copyright (c) 2020 Fayuan $\mathrm{Wu}$ et al. This is an open access article distributed under the Creative Commons Attribution License, which permits unrestricted use, distribution, and reproduction in any medium, provided the original work is properly cited.

\begin{abstract}
This work investigates the asynchronous control for fuzzy Markov switching systems (MSSs) with randomly occurring fading channel. By resorting to a T-S fuzzy model, the nonlinear MSSs can be handled. Meanwhile, in the unreliable network, the Rice fading model is proposed to capture the randomly occurring channel fading, which covers packet dropouts and network-induced delays as special cases. In light of the hidden Markov model, the asynchronous phenomenon of controller is taken into consideration, and asynchronous fuzzy controller is obtained. In the end, a numerical example and a single-link robotic arm model are applied to demonstrate the validity of the derived results.
\end{abstract}

\section{Introduction}

In physical applications, such as power station monitoring systems, fire-fighting operating systems, and electric networks, the signals are transmitted through a shared wireless/ wired communication channel. The stability of the systems is weakened by uncontrollable accidents on some occasions and external complex environment, and it is of importance to make the systems have certain robustness. However, because of the limited resources, the communication quality and computing load are affected, and the unexpected phenomenon occurs, such as networked-induced delay, quantization, and fading channel (FC). Normally, when signals are transmitted by wireless communication link, the phenomena of reflection/diffraction can be encountered, which results in multichannel-based fading. Recently, many scholars have devoted their attention to hybrid systems with FC [1-3]. It is remarkable that, compared with packet dropouts, FC is more general, which contains the finite coefficients. Lately, FC is widely applied in time-dependent probabilistic process, and many valuable results are reported in robust control [4], sliding mode control [5], and $H_{\infty}$ filtering $[6,7]$.

As a special type of switching systems, Markov switching systems (MSSs) consist of finite subsystems, and different subsystems are activated by a random Markov process [8-10]. Note that MSSs have been successfully applied in practical systems with probabilistic parameter changes, for instance, component failures. Added by its powerful application, many fruitful results have been gained in the issues of admissibility and stabilization, robust control, and filtering [11-14]. As one can be seen from [11-14] that, the control designed scheme has major concerns on synchronous situation. Owing to the networked mechanism and time delay, it is unreasonable to consider the synchronous scenario. By resorting to the hidden Markov model (HMM) [15-17], the asynchronous controller/filter can be solved in an application perspective.

On the contrary, it can be observed that aforementioned literatures are constrained to linear systems, which limited the practical application [18-23]. In general, MSSs tend to nonlinearities for external nonlinear environments and 
other factors, and it is urgent to take nonlinear MSSs into consideration [24-27]. Following this trend, many efforts have been devoted to nonlinear systems. Among them, Takagi-Sugeno (T-S) fuzzy model has been proved to be an efficient tool in tacking with nonlinear systems, in which by resorting to fuzzy inference strategy, the nonlinear systems are separated into finite local linear parts [28-30]. Recently, many meaningful results of T-S fuzzy MSSs (FMSSs) have been expressed in [20-22]. However, the FC issue has not been extended to FMSSs, not to mention asynchronous scenario.

Inspired by the above observation, we focus on the asynchronous control for FMSSs with randomly occurring FC. The major contributions are summarized as follows: (1) the FC model with disturbance is absorbed, which covers packet dropouts and network-induced delays as special cases; (2) added by parallel distributed compensation strategy and HMM scheme, the asynchronous control law is developed, which relaxes the limitation in existing results; and (3) by means of Lyapunov functional and stochastic analysis, sufficient criteria are gained and SLRAM is applied to illustrate the effectiveness of the proposed asynchronous control law.

The rest of this study is organized as below. The system descriptions are given in Section 2, and main results including stochastic stability of closed-loop system and controller gains are provided in Section 3. Computational experiments are expressed in Section 4. In the end, conclusions are shown in Section 5.

Notations: the notations utilized in this work are same as that in [10]. Furthermore, $\operatorname{sym}(L)=L+L^{\top}$ and $\operatorname{diag}\{\cdot\}$ means the block-diagonal matrix.

\section{Problem Formulations}

Consider the T-S FMSS (1) as follows:

Plant rule $i$ : IF $\phi_{1 k}$ is $M_{i 1}, \ldots, \phi_{f k}$ is $M_{i f}$, THEN

$$
\begin{aligned}
\delta(k+1) & =A\left(i, r_{k}\right) \delta(k)+B\left(i, r_{k}\right) u(k)+C\left(i, r_{k}\right) \omega(k), \\
z(k) & =D\left(i, r_{k}\right) \delta(k)+F\left(i, r_{k}\right) \omega(k),
\end{aligned}
$$

where $x(k) \in \mathbb{R}^{n_{x}}$ is the state vector, $u(k) \in \mathbb{R}^{n_{u}}$ is the control input, $z(k) \in \mathbb{R}^{n_{z}}$ is the output vector, and $\omega(k) \in \mathbb{R}^{n_{\omega}}$ is the external disturbance. Stochastic variable (SV) $r_{k}$ is a discrete Markov chain (DMC) and $r_{k} \in \mathscr{R}=\{1,2, \ldots, R\}$. For any $r_{k} \in \mathscr{R}, A_{r_{k}}, B_{r_{k}}, C_{r_{k}}, D_{r_{k}}$, and $F_{r_{k}}$ indicate the predetermined matrices. $M_{i j}$ are the fuzzy sets, and $\phi_{l k} \in\left\{\phi_{1 k}, \phi_{2 k}, \ldots, \phi_{f k}\right\}$ means the premise variable. Here, $i \in\{1,2, \ldots, \kappa\}$ and $\kappa$ implies the IF-THEN rules number.

In (1), for any $\mu, v \in \mathscr{R}$, the transition probability matrix (TPM) of original state is inferred by $\Gamma=\left[\vartheta_{\mu \nu}\right]$ :

$$
\operatorname{Pr}\left\{r_{k+1}=\nu \mid r_{k}=\mu\right\}=\vartheta_{\mu \nu},
$$

where $\mu, \nu \in \mathscr{R}, \vartheta_{\mu \nu} \in[0,1]$, and $\forall \mu \in \mathscr{R}, \sum_{\nu \in \mathscr{R}} \vartheta_{\mu \nu}=1$. For $r_{k}=\mu$, one has $\mathscr{R}\left(i, r_{k}\right)=\mathscr{R}_{i \mu}$, where $\mathscr{R}\left(i, r_{k}\right)=$ $\left\{A\left(i, r_{k}\right), B\left(i, r_{k}\right), C\left(i, r_{k}\right), D\left(i, r_{k}\right), F\left(i, r_{k}\right)\right\}$.
By means of the T-S fuzzy model, FMSS (1) can be deduced as follows:

$$
\begin{aligned}
\delta(k+1) & =\sum_{i=1}^{\kappa}\left(\hbar_{i}\left(\eta_{k}\right)\right)\left(A_{i \mu} \delta(k)+B_{i \mu} u(k)+C_{i \mu} \omega(k)\right), \\
z(k) & =\sum_{i=1}^{\kappa}\left(\hbar_{i}\left(\eta_{k}\right)\right)\left(D_{i \mu} \delta(k)+F_{i \mu} \omega(k)\right),
\end{aligned}
$$

where $M_{i j}\left(\eta_{j k}\right)$ symbolizes the grade of membership of $\eta_{j k}$ in $M_{i j}, \quad \Im_{i}\left(\eta_{k}\right)=\prod_{j=1}^{\kappa}\left(M_{i j}\left(\eta_{j k}\right)\right)$, and $\hbar_{i}\left(\eta_{k}\right)=$ $\mathfrak{\Im}_{i}\left(\eta_{k}\right) / \sum_{i=1}^{\kappa}\left(\mathfrak{\Im}_{i}\left(\eta_{k}\right)\right)$; here, $\hbar_{i}\left(\eta_{k}\right) \geq 0$, and $\sum_{i=1}^{\kappa} \hbar_{i}\left(\eta_{k}\right)=1$. For simplification, denoting

$$
\begin{aligned}
& A_{\hbar \mu}=\sum_{i=1}^{\kappa} \hbar_{i}\left(\eta_{k}\right) A_{i \mu}, \\
& B_{\hbar \mu}=\sum_{i=1}^{\kappa} \hbar_{i}\left(\eta_{k}\right) B_{i \mu}, \\
& C_{\hbar \mu}=\sum_{i=1}^{\kappa} \hbar_{i}\left(\eta_{k}\right) C_{i \mu}, \\
& D_{\hbar \mu}=\sum_{i=1}^{\kappa} \hbar_{i}\left(\eta_{k}\right) D_{i \mu}, \\
& F_{\hbar \mu}=\sum_{i=1}^{\kappa} \hbar_{i}\left(\eta_{k}\right) F_{i \mu},
\end{aligned}
$$

apparently, the FMSS (3) can be rewritten as

$$
\begin{aligned}
\delta(k+1) & =A_{\hbar \mu} \delta(k)+B_{\hbar \mu} u(k)+C_{\hbar \mu} \omega(k), \\
z(k) & =D_{\hbar \mu} \delta(k)+F_{\hbar \mu} \omega(k) .
\end{aligned}
$$

It is remarkable that the signals $\delta(k)$ are sending out via unreliable network; some unpredictable factors occur, such as channel fading (CF) and packet dropout. To model the randomly occurring $C F$, $L$ th-order $C F$ model is proposed:

$$
\bar{\delta}(k)=\sum_{l=0}^{L}\left(\alpha_{l}(k)\right) \delta(k-l)+H \zeta(k),
$$

where $\bar{\delta}(k)$ is the signal arrived at controller and $\alpha_{l}(k)(l=$ $0,1, \ldots, L)$ are mutually independent SVs with $\varepsilon\left\{\alpha_{l}(k)\right\}=\alpha_{l}$ subject to variances $\varepsilon\left\{\left(\alpha_{l}(k)-\alpha_{l}\right)^{2}\right\}=\bar{\alpha}_{l}^{2}$. $\zeta(k)$ represents the external disturbance and $H$ is the known matrix.

Remark 1. In the unreliable network, aiming to depict the unexpected networked phenomenon including scattering and other factors, fading channel is proposed, which is characterized by the Rice fading model. The stochastic variables $\alpha_{l}(k)(l=1,2, \ldots, L)$ can describe the variety of fading channel, which are applied and make the signal transmission more general.

In this work, by considering the unreliable of network medium, one establishes the following asynchronous control law (ACL):

Controller rule $i$ : IF $\varphi_{n k}$ is Min, $n \in(l=1,2, \ldots, f)$, THEN 


$$
u(k)=K_{i \tau_{k}} \bar{\delta}(k),
$$

where $K_{i \tau_{k}}$ indicates the unsolved controller gains. The SV $\tau_{k}$ is another DMC. DMC $\tau_{k} \in \mathscr{T}=\{1,2, \ldots, T\}$ represents HMM and obeys the conditional probability matrix CPM $\Lambda=\left[\varphi_{\mu t}\right]$ :

$$
\operatorname{Pr}\left\{\tau_{k}=t \mid r_{k}=\mu\right\}=\varphi_{\mu t},
$$

where $\varphi_{\mu t} \in[0,1]$ and $\sum_{t \in \mathscr{T}} \varphi_{\mu t}=1$. Accordingly, the ACL (7) is inferred as

$$
u(k)=K_{\hbar t} \bar{\delta}(k)
$$

where $K_{\hbar t}=\sum_{i=1}^{\kappa}\left(\hbar_{i}\left(\eta_{k}\right)\right) K_{i t}$.

Remark 2. Note that asynchronous phenomenon comes from the network-induced delay, quantization, and other factors, which results in unreasonable of traditional synchronous control law $[17,31]$. To tackle with such shortage, HMM is employed in designing controller, which is elaborated by $\left(r_{k}, \tau_{k}, \Gamma, \Lambda\right)$.

Substituting (6) and (9) into (5), the closed-loop FMSS can be acquired:

$$
\begin{aligned}
\delta(k+1)= & \left(A_{\hbar \mu}+\alpha_{0} B_{\hbar \mu} K_{\hbar t}\right) \delta(k)+C_{\hbar \mu} \omega(k) \\
& +\sum_{l=1}^{L} \alpha_{l} B_{\hbar \mu} K_{\hbar t} \delta(k-l)+B_{\hbar \mu} K_{\hbar t} H \zeta(k) \\
& +\widetilde{\alpha}_{0}(k) B_{\hbar \mu} K_{\hbar t} \delta(k)+\sum_{l=1}^{L} \tilde{\alpha}_{l}(k) B_{\hbar \mu} K_{\hbar t} \delta(k-l), \\
z(k)= & D_{\hbar \mu} \delta(k)+F_{\hbar \mu} \omega(k),
\end{aligned}
$$

where $\widetilde{\alpha}_{l}(k)=\alpha_{l}-\alpha_{l}(k)$.

Definition 1 (see [12]). Under the initial conditions $\delta(0), r_{0}$, the FMSS (10) is called stochastically stable (SS), such that

$$
\mathrm{E}\left\{\sum_{k=0}^{\infty}\|\delta(k)\|^{2} \mid \delta(0), r_{0}\right\}<\infty .
$$

Definition 2 (see [12]). The FMSS (10) is SS with $\mathscr{H}_{\infty}$ performance $\gamma$ such that

$$
\sum_{k=0}^{\infty}\|z(k)\|^{2}<\gamma^{2} \sum_{k=0}^{\infty} \omega^{\top}(k) \omega(k) .
$$

\section{Main Results}

Theorem 1. If there exists a scalar $\gamma>0$, matrices $P_{\mu}>0(\mu \in \mathscr{R}), \quad U_{\mu t}>0(\mu \in \mathscr{R}, t \in \mathscr{T})$,
$Q_{l}>0(l=1,2, \ldots, L)$, the FMSS (10) is SS with $\mathscr{H}_{\infty}$ performance $\gamma$, for any $\mu, \nu \in \mathscr{R}, t \in \mathscr{T}$, such that

$$
\begin{array}{r}
\sum_{t \in \mathscr{T}} \varphi_{\mu t} U_{\mu t}<P_{\mu}, \\
\Omega_{i i \mu t}<0, \\
\Omega_{i j \mu t}+\Omega_{j i \mu t}<0,
\end{array}
$$

where

$$
\begin{aligned}
\Omega_{i j \mu t} & =\left[\begin{array}{cc}
\Omega_{i j \mu t}^{1} & \Omega_{i j \mu t}^{2} \\
& \Omega_{\mu}^{3}
\end{array}\right], \\
\mathscr{P}_{\mu} & =\sum_{\nu \in \mathscr{R}} \vartheta_{\mu \nu} P_{\nu}, \\
\Omega_{i j \mu t}^{1} & =\operatorname{diag}\left\{\Omega_{i j \mu t}^{11}, \Omega_{i j \mu t}^{12}, \Omega_{i j \mu t}^{13}, \Omega_{i j \mu t}^{14}\right\}, \\
\Omega_{i j \mu t}^{2} & =\left[\Omega_{i j \mu t}^{21} \Omega_{i j \mu t}^{22} \Omega_{i j \mu t}^{23} \Omega_{i j \mu t}^{24}\right], \\
\Omega_{\mu}^{3} & =\operatorname{diag}\left\{-\mathscr{P}_{\mu},-\mathscr{P}_{\mu},-I_{L} \otimes \mathscr{P}_{\mu},-I\right\}, \\
\Omega_{i j \mu t}^{11} & =-U_{\mu t}+\sum_{q=1}^{L} Q_{q}, \\
\Omega_{i j \mu t}^{13} & =-\gamma^{2} I, \\
\Omega_{i j \mu t}^{12} & =\operatorname{diag}-Q_{1},-Q_{2}, \ldots,-Q_{L}, \\
\Omega_{i j \mu t}^{14} & =-\gamma^{2} I,
\end{aligned}
$$$$
\Omega_{i j \mu t}^{21}=\left[\begin{array}{c}
\left(A_{i \mu}+\alpha_{0} B_{i \mu} K_{j t}\right)^{\top} \mathscr{P}_{\mu} \\
\left(B_{j \mu} K_{j t} \mathscr{Z}\right)^{\top} \mathscr{P}_{\mu} \\
C_{i \mu}^{\top} \mathscr{P}_{\mu} \\
\left(B_{i \mu} K_{j t} H\right)^{\top} \mathscr{P}_{\mu}
\end{array}\right] \text {, }
$$$$
\Omega_{i j \mu t}^{22}=\left[\begin{array}{c}
\bar{\alpha}_{0}\left(B_{i \mu} K_{j t}\right)^{\top} \mathscr{P}_{\mu} \\
0 \\
0 \\
0
\end{array}\right] \text {, }
$$$$
\mathscr{Z}=\left[\alpha_{1} I \alpha_{2} I \cdots \alpha_{L} I\right] \text {, }
$$$$
\Omega_{i j \mu t}^{23}=\left[\begin{array}{c}
0 \\
\left(\bar{\Theta} \otimes B_{i \mu} K_{j t}\right)^{\top} \mathscr{P}_{\mu} \\
0 \\
0
\end{array}\right] \text {, }
$$$$
\Omega_{i j \mu t}^{24}=\left[\begin{array}{llll}
D_{i \mu} & 0 & F_{i \mu} 0
\end{array}\right]^{\top} \text {, }
$$$$
\bar{\Theta}=\operatorname{diag}\left\{\bar{\alpha}_{1}, \bar{\alpha}_{2}, \ldots, \bar{\alpha}_{L}\right\} \text {. }
$$

Proof. It follows from (14) and (15) that 


$$
\begin{aligned}
\Omega_{\hbar \mu t} & =\sum_{i=1}^{\kappa} \sum_{j=1}^{\kappa} \hbar_{i}\left(\eta_{k}\right) \hbar_{j}\left(\eta_{k}\right) \Omega_{i j \mu t} \\
& =\sum_{i=1}^{\kappa} \hbar_{i}^{2}\left(\eta_{k}\right) \Omega_{i i \mu t}+\sum_{i=1}^{\kappa-1} \sum_{j=i+1}^{\kappa} \hbar_{i}\left(\eta_{k}\right) \hbar_{j}\left(\eta_{k}\right)\left(\Omega_{i j \mu t}+\Omega_{j i \mu t}\right)<0,
\end{aligned}
$$

where

$$
\begin{aligned}
& \Omega_{\hbar \mu t}=\left[\begin{array}{cc}
\Omega_{\hbar \mu t}^{1} & \Omega_{\hbar \mu t}^{2} \\
& \Omega_{\mu}^{3}
\end{array}\right], \\
& \Omega_{\hbar \mu t}^{1}=\operatorname{diag}\left\{\Omega_{\hbar \mu t}^{11}, \Omega_{\hbar \mu t}^{12}, \Omega_{\hbar \mu t}^{13}, \Omega_{\hbar \mu t}^{14}\right\}, \\
& \Omega_{\hbar \mu t}^{2}=\left[\begin{array}{llll}
\Omega_{\hbar \mu t}^{21} & \Omega_{\hbar \mu t}^{22} & \Omega_{\hbar \mu t}^{23} & \Omega_{\hbar \mu t}^{24}
\end{array}\right], \\
& \Omega_{\hbar \mu}^{3}=\operatorname{diag}\left\{-p_{\mu},-p_{\mu},-I_{L} \otimes p_{\mu},-I\right\}, \\
& \Omega_{\hbar \mu t}^{11}=-P_{\mu}+\sum_{q=1}^{L} Q_{q}, \\
& \Omega_{\hbar \mu t}^{13}=-\gamma^{2} I, \\
& \Omega_{\hbar \mu t}^{12}=\operatorname{diag}\left\{-Q_{1},-Q_{2}, \ldots,-Q_{L}\right\}, \\
& \Omega_{\hbar \mu t}^{21}=\left[\begin{array}{c}
\left(A_{\hbar \mu}+\alpha_{0} B_{\hbar \mu} K_{\hbar t}\right)^{T} p_{\mu} \\
\left(B_{\hbar \mu} K_{\hbar t} Z\right)^{T} p_{\mu} \\
C_{\hbar \mu}^{T} p_{\mu} \\
\left(B_{\hbar \mu} K_{\hbar t} H\right)^{T} p_{\mu}
\end{array}\right] \text {, } \\
& \Omega_{\hbar \mu t}^{22}=\left[\begin{array}{c}
\bar{\alpha}_{0}\left(B_{\hbar \mu} K_{\hbar t}\right)^{T} p_{\mu} \\
0 \\
0 \\
0
\end{array}\right] \text {, } \\
& \Omega_{\hbar \mu t}^{14}=-\gamma^{2} I, \\
& \Omega_{\hbar \mu t}^{23}=\left[\begin{array}{c}
0 \\
\left(\bar{\Theta} \otimes B_{\hbar \mu} K_{\hbar t}\right)^{T} p_{\mu} \\
0 \\
0
\end{array}\right] \text {, } \\
& \Omega_{\hbar \mu t}^{24}=\left[\begin{array}{llll}
D_{\hbar \mu} & 0 & F_{\hbar \mu} & 0
\end{array}\right]^{T} \text {. }
\end{aligned}
$$

Construct the Lyapunov functional for FMSS (10):

$$
V(k, \delta(k))=V_{1}(k, \delta(k))+V_{2}(k, \delta(k)),
$$

where

$$
\begin{aligned}
& V_{1}(k, \delta(k))=\delta^{\top}(k) P_{\mu} \delta(k), \\
& V_{2}(k, \delta(k))=\sum_{q=1}^{L} \sum_{p=k-q}^{k-1} \delta^{\top}(p) Q_{p} \delta(p) .
\end{aligned}
$$

Calculating the difference of $V(k, \delta(k))$ and taking expectation, one has

$$
\mathrm{E}\{\Delta V(k)\}=\mathrm{E}\left\{V\left(k+1, \delta_{k+1}\right) \mid \delta(k), r_{k}\right\}-V(k, \delta(k))
$$

Along system (10), it yields that 


$$
\begin{aligned}
\varepsilon\left\{\Delta V_{1}(k)\right\}= & \varepsilon\left\{\delta^{T}(k+1) p_{\mu} \delta(k+1)-\delta^{T}(k) p_{\mu} \delta(k)\right\} \\
= & \varepsilon\left\{\left[\left(A_{\hbar \mu}+\alpha_{0} B_{\hbar \mu} K_{\hbar t}\right) \delta(k)+C_{\hbar \mu} \omega(k)+\sum_{l=1}^{L} \alpha_{l} B_{\hbar \mu} K_{\hbar t} \delta(k-l)+B_{\hbar \mu} K_{\hbar t} H \varsigma(k)\right]^{T}\right. \\
& \cdot p_{u}\left[\left(A_{\hbar \mu}+\alpha_{0} B_{\hbar \mu} K_{\hbar t}\right) \delta(k)+C_{\hbar \mu} \omega(k)+\sum_{l=1}^{L} \alpha_{l} B_{\hbar \mu} K_{\hbar t} \delta(k-1)+B_{\hbar \mu} K_{\hbar t} H \zeta(k)\right] \\
+ & \widetilde{\alpha}_{0}^{2}(k) \delta^{T}(k) K_{\hbar t}^{T} B_{\hbar \mu}^{T} p_{\mu} B_{\hbar \mu} K_{\hbar t} \delta(k)+\left(\sum_{l=1}^{L} \widetilde{\alpha}_{l}(k) B_{\hbar \mu} K_{\hbar t} \delta(k-l)\right)^{T} \\
& \left.\cdot p_{\mu} \times\left(\sum_{l=1}^{L} \widetilde{\alpha}_{l}(k) B_{\hbar \mu} K_{\hbar t} \delta(k-l)\right)\right\}-\delta^{T}(k) P_{\mu} \delta(k) .
\end{aligned}
$$

Added by the variances in fading channels $\bar{\alpha}_{l}(l=0,1, \ldots, L),(22)$ can be reformulated as

$$
\varepsilon\left\{\Delta V_{1}(k)\right\} \leq \varepsilon\left\{\begin{array}{l}
{\left[\left(A_{\hbar \mu}+\alpha_{0} B_{\hbar \mu} K_{\hbar t}\right) \delta(k)+C_{\hbar \mu} \omega(k)+B_{\hbar \mu} K_{\hbar t} \mathscr{E} \delta_{L}(k)+B_{\hbar \mu} K_{\hbar t} H \zeta(k)\right]^{\top}} \\
\times \mathscr{P}_{\mu}\left[\left(A_{\hbar \mu}+\alpha_{0} B_{\hbar \mu} K_{\hbar t}\right) \delta(k)+C_{\hbar \mu} \omega(k)+B_{\hbar \mu} K_{\hbar t} \mathscr{Z} \delta_{L}(k)+B_{\hbar \mu} K_{\hbar t} H \zeta(k)\right] \\
+\bar{\alpha}_{0}^{2} \delta^{\top}(k) K_{\hbar t}^{\top} B_{\hbar \mu}^{\top} \mathscr{P}_{\mu} B_{\hbar \mu} K_{\hbar t} \delta(k)+\delta_{L}^{\top}(k)\left[\bar{\Theta}^{2} \otimes K_{\hbar t}^{\top} B_{\hbar \mu}^{\top} \mathscr{P}_{\mu} B_{\hbar \mu} K_{\hbar t}\right] \delta_{L}(k)
\end{array}\right\}-\delta^{\top}(k) P_{\mu} \delta(k),
$$

where

$$
\delta_{L}(k)=\left[\delta^{\top}(k-1) \delta^{\top}(k-2) \cdots \delta^{\top}(k-L)\right]^{\top} .
$$

$$
\begin{aligned}
\varepsilon\left\{\Delta V_{2}(k)\right\} & =\varepsilon\left\{\sum_{q=1}^{L} \sum_{p=k-q+1}^{k} \delta^{\top}(p) Q_{p} \delta(p)-\sum_{q=1}^{L} \sum_{p=k-q}^{k-1} \delta^{\top}(p) Q_{p} \delta(p)\right\} \\
& =\delta^{\top}(k) \sum_{q=1}^{L} Q_{q} \delta(k)-\sum_{q=1}^{L} \delta^{\top}(k-q) Q_{q} \delta(k-q) .
\end{aligned}
$$

For simplification, denoting $₫(k)=\left[\omega^{\top}(k) \zeta^{\top}(k)\right]^{\top}$. Firstly, we will prove the SS of system (10) with $₫(k)=0$.

Combining with (23) and (25), one obtains

$$
\begin{aligned}
\mathcal{E}\{\Delta V(k)\} \leq \varepsilon & \left\{\begin{array}{c}
{\left[\left(A_{\hbar \mu}+\alpha_{0} B_{\hbar \mu} K_{\hbar t}\right) \delta(k)+B_{\hbar \mu} K_{\hbar t} \mathscr{E} \delta_{L}(k)\right]^{\top} \times \mathscr{P}_{\mu}\left[\left(A_{\hbar \mu}+\alpha_{0} B_{\hbar \mu} K_{\hbar t}\right) \delta(k)+B_{\hbar \mu} K_{\hbar t} \mathscr{E} \delta_{L}(k)\right]} \\
+\bar{\alpha}_{0}^{2} \delta^{\top}(k) K_{\hbar t}^{\top} B_{\hbar \mu}^{\top} \mathscr{P}_{\mu} B_{\hbar \mu} K_{\hbar t} \delta(k)+\delta_{L}^{\top}(k)\left[\bar{\Theta}^{2} \otimes K_{\hbar t}^{\top} B_{\hbar \mu}^{\top} \mathscr{P}_{\mu} B_{\hbar \mu} K_{\hbar t}\right] \delta_{L}(k)
\end{array}\right\} \\
& -\delta^{\top}(k) P_{\mu} \delta(k)+\delta^{\top}(k) \sum_{q=1}^{L} Q_{q} \delta(k)-\sum_{q=1}^{L} \delta^{\top}(k-q) Q_{q} \delta(k-q) .
\end{aligned}
$$


Applying Schur complement to (26), it is clear that

$$
\varepsilon\{\Delta V(k)\} \leq \varepsilon\left\{\widetilde{\psi}^{\top}(k) \widetilde{\Omega}_{\hbar \mu} \widetilde{\psi}(k)\right\},
$$

where

$$
\begin{aligned}
\widetilde{\psi}(k) & =\left[\delta^{\top}(k) \delta_{L}^{\top}(k)\right]^{\top}, \\
\widetilde{\Omega}_{\hbar \mu t} & =\left[\begin{array}{cc}
\widetilde{\Omega}_{\hbar \mu t}^{1} & \widetilde{\Omega}_{\hbar \mu t}^{2} \\
& \widetilde{\Omega}_{\mu}^{3}
\end{array}\right], \\
\widetilde{\Omega}_{\hbar \mu t}^{1} & =\operatorname{diag}\left\{\Omega_{\hbar \mu t}^{11}, \Omega_{\hbar \mu t}^{12}\right\}, \\
\widetilde{\Omega}_{\hbar \mu t}^{2} & =\left[\Omega_{\hbar \mu t}^{21} \Omega_{\hbar \mu t}^{22} \Omega_{\hbar \mu t}^{23}\right], \\
\widetilde{\Omega}_{\mu}^{3} & =\operatorname{diag}\left\{-\mathscr{P}_{\mu},-\mathscr{P}_{\mu},-\mathscr{P}_{\mu}\right\} .
\end{aligned}
$$

In light of (27), it achieves that

$$
\varepsilon\{\Delta V(k)\} \leq \rho \varepsilon\left\{\delta^{\top}(k) \delta(k)\right\},
$$

where $\rho=\lambda_{\max }\left\{\widetilde{\Omega}_{\hbar \mu t}\right\}, \quad(\forall i=1,2, \ldots, \kappa, \mu \in \mathscr{R}, t \in \mathscr{T})$. By (17), it is clear that $\rho<0$, which indicates

$$
\varepsilon\left\{\delta^{\top}(k) \delta(k)\right\} \leq \frac{1}{\rho} \varepsilon\{\Delta V(k)\} .
$$

For $N \longrightarrow \infty$, it yields

$$
\varepsilon\left\{\sum_{k=0}^{\infty} \delta^{\top}(k) \delta(k)\right\} \leq-\frac{1}{\rho} V(0, \delta(0))<\infty .
$$

By Definition 1, it is obviously that FMSS (10) is SS with $\varpi(k)=0$.

Next, the FMSS (10) is SS with the $\mathscr{H}_{\infty}$ performance index will be verified. For $₫(k) \neq 0$ under zero-initial condition, denoting $\mathscr{J}(k)=\sum_{k=0}^{\infty} \varepsilon\left\{\|z(k)\|^{2}\right\}-\gamma^{2} \varepsilon\left\{\|\varpi(k)\|^{2}\right\}$, one has

$$
\begin{aligned}
\mathscr{J}(k) \leq & \sum_{k=0}^{\infty} \varepsilon\left\{z^{T}(k) z(k)-\gamma^{2} \varpi^{T}(k) \varpi(k)+\Delta V(k)\right\} \\
\leq & \sum_{k=0}^{\infty} \varepsilon\left\{\left[\left(A_{\hbar \mu}+\alpha_{0} B_{\hbar \mu} K_{\hbar t}\right) \delta(k)+C_{\hbar \mu} \omega(k)+B_{\hbar \mu} K_{\hbar t} Z \delta_{L}(k)+B_{\hbar \mu} K_{\hbar t} H \zeta(k)\right]^{T}\right. \\
& \times P_{\mu}\left[\left(A_{\hbar \mu}+\alpha_{0} B_{\hbar \mu} K_{\hbar t}\right) \delta(k)+C_{\hbar \mu} \omega(k)+B_{\hbar \mu} K_{\hbar t} Z \delta_{L}(k)+B_{\hbar \mu} K_{\hbar t} H \zeta(k)\right] \\
& +\bar{\alpha}_{0}^{2} \delta^{T}(k) K_{\hbar t}^{T} B_{\hbar t}^{T} P_{\mu} B_{\hbar \mu} K_{\hbar t} \delta(k)+\delta_{L}^{T}(k)\left[\bar{\Theta}^{2} \otimes K_{\hbar \mu}^{T} B_{\hbar \mu}^{T} P_{\mu} B_{\hbar \mu} K_{\hbar t}\right] \delta_{L}(k) \\
& -\delta^{T}(k) P_{\mu} \delta(k)+\delta^{T}(k) \sum_{q=1}^{L} Q_{q} \delta(k)-\sum_{q=1}^{L} \delta^{T}(k-q) Q_{q} \delta(k-q) \\
& \left.+\left[D_{\hbar \mu} \delta(k)+F_{\hbar \mu} \omega(k)\right]^{T}\left[D_{\hbar \mu} \delta(k)+F_{\hbar \mu} \omega(k)\right]-\gamma^{2} \omega^{T}(k) \oplus(k)\right\} .
\end{aligned}
$$

Deploy Schur complement to (32), which results in

$$
\mathscr{J}(k) \leq \sum_{k=0}^{\infty}\left\{\psi^{\top}(k) \Omega_{\hbar \mu} \psi(k)\right\},
$$

where $\psi(k)=\left[\delta^{\top}(k) \delta_{L}^{\top}(k) \omega^{\top}(k) \zeta^{\top}(k)\right]^{\top}$.

In light of (17) and (33), it can be concluded that

$$
\lim _{N \longrightarrow \infty}\left\{\left\{\sum_{k=0}^{N} z^{\top}(k) z(k)\right\}<\gamma^{2} \sum_{k=0}^{N} \omega^{\top}(k) \omega(k),\right.
$$

which indicates $\varepsilon\left\{\sum_{k=0}^{\infty} z^{\top}(k) z(k)\right\}<\gamma^{2} \sum_{k=0}^{\infty} \omega^{\top}(k) \omega(k)$. By Definition 2, the FMSS (10) is SS with $\mathscr{H}_{\infty}$ performance $\gamma$.
Theorem 2. If there exists a scalar $\gamma>0$, matrices $P_{\mu}>0(\mu \in \mathscr{R}), \quad U_{\mu t}>0(\mu \in \mathscr{R}, t \in \mathscr{T}), \quad Q_{l}>0(l=1,2$, $\ldots, L)$, and matrices $W_{t}(t \in \mathscr{T}), \quad Y_{t}(t \in \mathscr{T})$, $\bar{K}_{j t}(j=1,2, \ldots, \kappa, t \in \mathscr{T})$, the FMSS (10) is SS with $\mathscr{H}_{\infty}$ performance $\gamma$, for any $\mu, v \in \mathscr{R}, t \in \mathscr{T}$, such that

$$
\begin{gathered}
W_{t} B_{i \mu}=B_{i \mu} Y_{t}, \\
\Upsilon_{i i \mu t}<0, \\
\Upsilon_{i j \mu t}+\Upsilon_{j i \mu t}<0,
\end{gathered}
$$

where 


$$
\begin{aligned}
& \Upsilon_{i j \mu t}=\left[\begin{array}{ll}
\Omega_{i j \mu t}^{1} & \Upsilon_{i j \mu t}^{2} \\
& \Upsilon_{\mu t}^{3}
\end{array}\right], \\
& \Upsilon_{i j \mu t}^{2}=\left[\begin{array}{llll}
\Upsilon_{i j \mu t}^{21} & \Upsilon_{i j \mu t}^{22} & \Upsilon_{i j \mu t}^{23} & \Upsilon_{i j \mu t}^{24}
\end{array}\right] \text {, } \\
& \Upsilon_{i j \mu t}^{21}=\left[\begin{array}{c}
A_{i \mu}^{T} W_{t}^{T}+\alpha_{0} \bar{K}_{j t}^{T} B_{i \mu}^{T} \\
z^{T} \bar{K}_{j t}^{T} B_{i \mu}^{T} \\
C_{i \mu}^{T} W_{t}^{T} \\
H^{T} \bar{K}_{j t}^{T} B_{i \mu}^{T}
\end{array}\right], \\
& \Upsilon_{i j \mu t}^{22}=\left[\begin{array}{c}
\bar{\alpha}_{0} \bar{K}_{j t}^{T} B_{i \mu}^{T} \\
0 \\
0 \\
0
\end{array}\right] \text {, } \\
& \Upsilon_{i j \mu t}^{23}\left[\begin{array}{c}
0 \\
\Theta \otimes\left(\bar{K}_{j t}^{T} B_{i \mu}^{T}\right) \\
0 \\
0
\end{array}\right], \\
& \Upsilon_{\mu t}^{3}=\operatorname{diag}\left\{\mathscr{P}_{\mu}-\operatorname{sym}\left\{W_{t}\right\}, \mathscr{P}_{\mu}-\operatorname{sym}\left\{W_{t}\right\},\right. \\
& \left.I_{L} \otimes\left(\mathscr{P}_{\mu}-\operatorname{sym}\left\{W_{t}\right\}\right),-I\right\} \text {. }
\end{aligned}
$$

The controller parameters can be obtained by

$$
K_{j t}=Y_{t}^{-1} \bar{K}_{j t}, \quad(j=1,2, \ldots, \kappa, t \in \mathscr{T}) .
$$

Proof. Define

$$
\mathscr{M}_{\mu t}=\operatorname{diag}\left\{I, I, \ldots, I, \mathscr{P}_{\mu}^{-1} W_{t}^{\top}, \mathscr{P}_{\mu}^{-1} W_{t}^{\top}, I_{L} \otimes \mathscr{P}_{\mu}^{-1} W_{t}^{\top}, I\right\} .
$$
that

Applying a congruence transformation to (14), it yields

$$
\bar{\Upsilon}_{i i \mu t}=\mathscr{M}_{\mu t}^{\top} \Omega_{i i \mu t} \mathscr{M}_{\mu t}<0,
$$

where

$$
\begin{aligned}
\bar{\Upsilon}_{i i \mu t}= & {\left[\begin{array}{cc}
\Omega_{i i \mu t}^{1} & \bar{\Upsilon}_{i i \mu t}^{2} \\
\bar{\Upsilon}_{\mu t}^{3}
\end{array}\right], } \\
\bar{\Upsilon}_{i i \mu t}^{2}= & {\left[\begin{array}{lc}
\bar{\Upsilon}_{i i \mu t}^{21} & \bar{\Upsilon}_{i i \mu t}^{22} \bar{\Upsilon}_{i i \mu t}^{23} \Omega_{i i \mu t}^{24}
\end{array}\right], } \\
\bar{\Upsilon}_{i i \mu t}^{21}= & {\left[\begin{array}{c}
A_{i \mu}^{\top} W_{t}^{\top}+\alpha_{0} \bar{K}_{i t}^{\top} B_{i \mu}^{\top} \\
\mathscr{Z}^{\top} K_{i t}^{\top} B_{i \mu}^{\top} W_{t}^{\top} \\
C_{i \mu}^{\top} W_{t}^{\top} \\
H^{\top} K_{i t}^{\top} B_{i \mu}^{\top} W_{t}^{\top}
\end{array}\right], } \\
\bar{\Upsilon}_{i i \mu t}^{22}= & {\left[\begin{array}{c}
\bar{\alpha}_{0} K_{i t}^{\top} B_{i \mu}^{\top} W_{t}^{\top} \\
0 \\
0 \\
0
\end{array}\right], } \\
\bar{\Upsilon}_{\mu t}^{3}= & \operatorname{diag}\left\{-W_{t} \mathscr{P}_{\mu}^{-1} W_{t}^{\top},-W_{t} \mathscr{P}_{\mu}^{-1} W_{t}^{\top},\right. \\
\bar{\Upsilon}_{i i \mu t}^{23}= & {\left[\begin{array}{c}
\left.I_{L} \otimes\left(W_{t} \mathscr{P}_{\mu}^{-1} W_{t}^{\top}\right),-I\right\} . \\
\Theta \otimes\left(K_{i t}^{\top} B_{i \mu}^{\top} W_{t}^{\top}\right) \\
0 \\
0
\end{array}\right], }
\end{aligned}
$$

From $\left(\mathscr{P}_{\mu}-W_{t}\right) \mathscr{P}_{\mu}^{-1}\left(\mathscr{P}_{\mu}-W_{t}^{\top}\right)>0$, it follows that

$$
-W_{t} \mathscr{P}_{\mu}^{-1} W_{t}^{\top}<\mathscr{P}_{\mu}-\operatorname{sym}\left\{W_{t}\right\} .
$$

By resorting to (35), (39), and (43), (35) can be guaranteed if (14) holds. By the same way, (37) and (39) can be guaranteed if (15) and (17) hold.

\section{Computational Experiments}

4.1. Numerical Example. Consider the FMSS (1) with 3 modes, its parameters are chosen as follows: 
$A_{11}=\left[\begin{array}{cc}-0.7933 & -0.0250 \\ -0.4065 & 0.4676\end{array}\right]$,

$B_{11}=\left[\begin{array}{c}-1.3569 \\ 1.6143\end{array}\right]$,

$C_{11}=\left[\begin{array}{l}-0.2241 \\ -0.1587\end{array}\right]$,

$D_{11}=\left[\begin{array}{ll}1.2622 & -0.3065\end{array}\right]$,

$A_{12}=\left[\begin{array}{cc}0.5916 & -1.5784 \\ -0.6035 & 0.1609\end{array}\right]$,

$B_{12}=\left[\begin{array}{c}-0.3191 \\ 0.9530\end{array}\right]$,

$C_{12}=\left[\begin{array}{c}-1.4349 \\ 0.9430\end{array}\right]$,

$D_{12}=\left[\begin{array}{ll}-0.4898 & 0.6855\end{array}\right]$,

$A_{13}=\left[\begin{array}{cc}0.6255 & 0.0520 \\ 0.2561 & -0.7916\end{array}\right]$,

$B_{13}=\left[\begin{array}{l}0.7225 \\ 0.2704\end{array}\right]$,

$C_{13}=\left[\begin{array}{c}1.4089 \\ -0.4147\end{array}\right]$,

$D_{13}=\left[\begin{array}{ll}1.0335 & 2.2099\end{array}\right]$,

$A_{21}=\left[\begin{array}{ll}-0.3922 & -0.0717 \\ -0.4506 & -0.0868\end{array}\right]$,

$B_{21}=\left[\begin{array}{c}-0.6664 \\ 0.5477\end{array}\right]$,

$C_{21}=\left[\begin{array}{c}-0.0816 \\ 0.3037\end{array}\right]$,

$D_{21}=\left[\begin{array}{ll}-0.3504 & 2.6857\end{array}\right]$,

$A_{22}=\left[\begin{array}{cc}0.8048 & 0.5603 \\ 0.6455 & -0.6569\end{array}\right]$,

$B_{22}=\left[\begin{array}{c}0.5714 \\ -1.1684\end{array}\right]$,

$C_{22}=\left[\begin{array}{l}1.9891 \\ 0.8228\end{array}\right]$,

$D_{22}=\left[\begin{array}{ll}-2.0949 & 0.4153\end{array}\right]$,

$A_{23}=\left[\begin{array}{cc}-0.3107 & 0.0627 \\ 0.4732 & 0.7070\end{array}\right]$,

$B_{23}=\left[\begin{array}{c}0.0078 \\ -0.8450\end{array}\right]$,

$C_{23}=\left[\begin{array}{l}0.0112 \\ 1.0132\end{array}\right]$,

$D_{23}=\left[\begin{array}{ll}-0.0921-0.5206\end{array}\right]$,

$F_{i \mu}=0.01, \quad(i=1,2, \mu=1,2,3)$,

$H=\left[\begin{array}{ll}0.05 & 0.05\end{array}\right]^{T}$.
The TPM $\Gamma$ and $\mathrm{CPM} \Lambda$ are inferred as

$$
\begin{aligned}
\Gamma & =\left[\begin{array}{lll}
0.4 & 0.5 & 0.1 \\
0.05 & 0.05 & 0.9 \\
0.2 & 0.4 & 0.4
\end{array}\right], \\
\Lambda & =\left[\begin{array}{ll}
0.8 & 0.2 \\
0.5 & 0.5 \\
0.7 & 0.3
\end{array}\right] .
\end{aligned}
$$

One assumes that the Lth-order of CF model (6) with $L=1$ and $\alpha_{0}=0.78, \alpha_{1}=0.36, \bar{\alpha}_{0}=0.3$, and $\bar{\alpha}_{1}=0.3$. $\omega(k)=\left(1.6 \sin ^{2}(k)\right) / 1+k^{2}, \zeta(k)=0.8 \exp \left(-k^{2}\right)$. By solving the inequalities of Theorem 2, a set of controller gains are acquired:

$$
\left[\begin{array}{ll}
K_{11} & K_{12} \\
K_{21} & K_{22}
\end{array}\right]=\left[\begin{array}{cccc}
-0.1301 & -0.1029 & -0.1329 & -0.1061 \\
0.1855 & 0.1442 & 0.1709 & 0.1330
\end{array}\right] .
$$

By applying the aforementioned controller gains, Figures 1 and 2 demonstrate the state mode and controller mode, respectively. The state trajectories with CF are shown in Figure 3, and the control input is depicted in Figure 4.

Defining $\gamma_{d}(k)=\sqrt{\sum_{s=0}^{k}\|z(s)\|^{2} / \sum_{s=0}^{k}\|\varpi(s)\|^{2}}$, Figure 5 plots the evolution of $\gamma_{d}(k)$.

4.2. Practical Example. Robot arms are essential parts in firefighting operating systems. In this case, we consider a singlelink robotic arm model (SLRAM) stemmed from [12], which is inferred by

$$
\ddot{\phi}(t)=-\frac{g \mathscr{L} \mathscr{M}_{k}}{\mathscr{J}_{k}} \sin (\phi(t))-\frac{\mathscr{R}}{\mathscr{J}_{k}} \dot{\phi}(t)+\frac{1}{\mathscr{J}_{k}} u(t)+\omega(t),
$$

where the meaning of $g, \phi(t), \mathscr{L}, \mathscr{J}_{k}$, and $\mathscr{R}$ are explained in [12] and $g=9.81 \mathrm{~m} / \mathrm{s}^{2}, \quad \mathscr{L}=0.5 \mathrm{~m}, \quad \mathscr{R}=1 \mathrm{~kg} \cdot \mathrm{m}^{2} / \mathrm{s}$, $\mathscr{M}_{1}=1 \mathrm{~kg}, \mathscr{M}_{2}=5 \mathrm{~kg}, \mathscr{J}_{1}=1 \mathrm{~kg} \cdot \mathrm{m}^{2}$, and $\mathscr{J}_{2}=5 \mathrm{~kg} \cdot \mathrm{m}^{2}$.

According to [12], for sampling period $\mathscr{T}=0.01 \mathrm{~s}$, the SLRAM is reestablished as the T-S FMSSs.

Plant rule 1: IF $\delta_{1}(k)$ is about $0 \mathrm{rad}$, THEN

$$
\begin{aligned}
\delta(k+1) & =A_{1, \mu} \delta(k)+B_{1, \mu} u(k)+C_{1, \mu} \omega(k), \\
z(k) & =D_{1, \mu} \delta(k)+F_{1, \mu} \omega(k) .
\end{aligned}
$$

Plant rule 2: IF $\delta_{2}(k)$ is about $\pm \pi \operatorname{rad}$, THEN

$$
\begin{aligned}
\delta(k+1) & =A_{2, \mu} \delta(k)+B_{2, \mu} u(k)+C_{2, \mu} \omega(k), \\
z(k) & =D_{2, \mu} \delta(k)+F_{2, \mu} \omega(k),
\end{aligned}
$$

where 


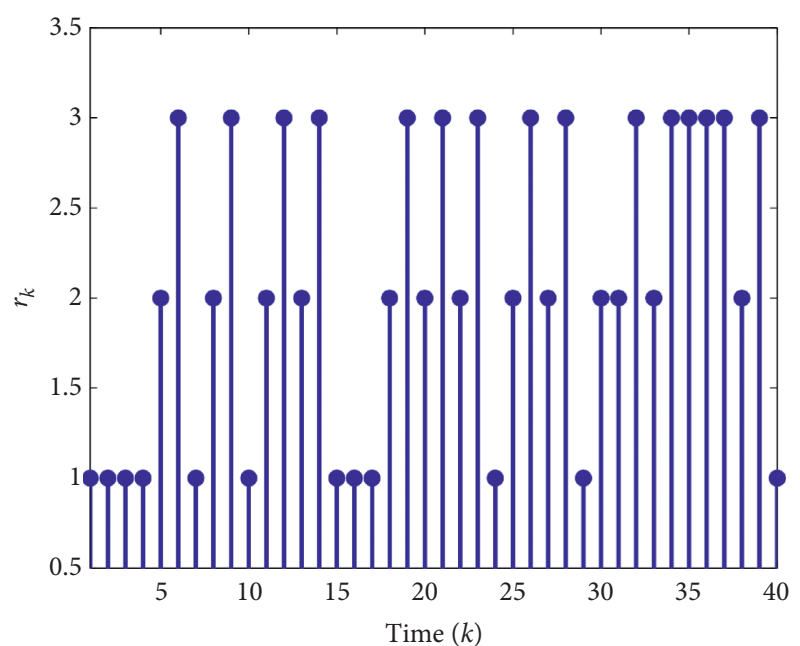

Figure 1: State mode.

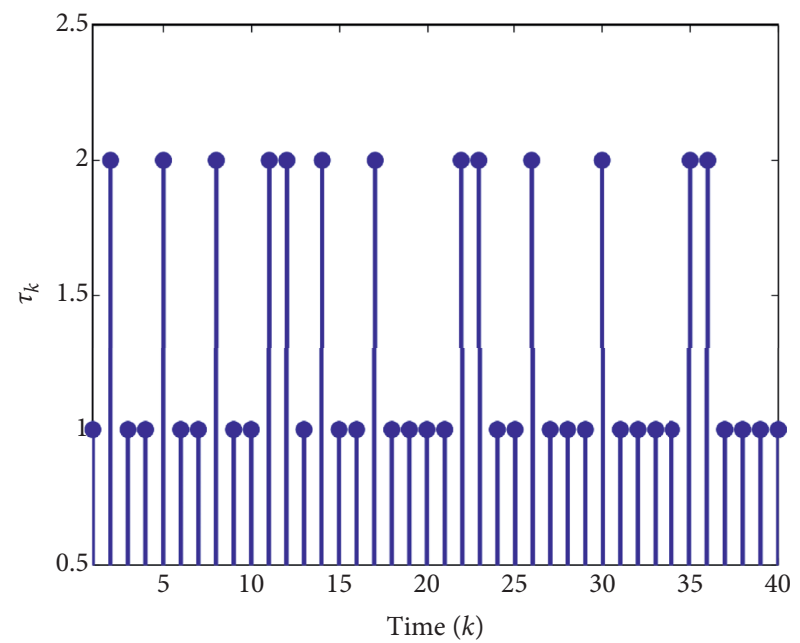

FIgURE 2: Controller mode.

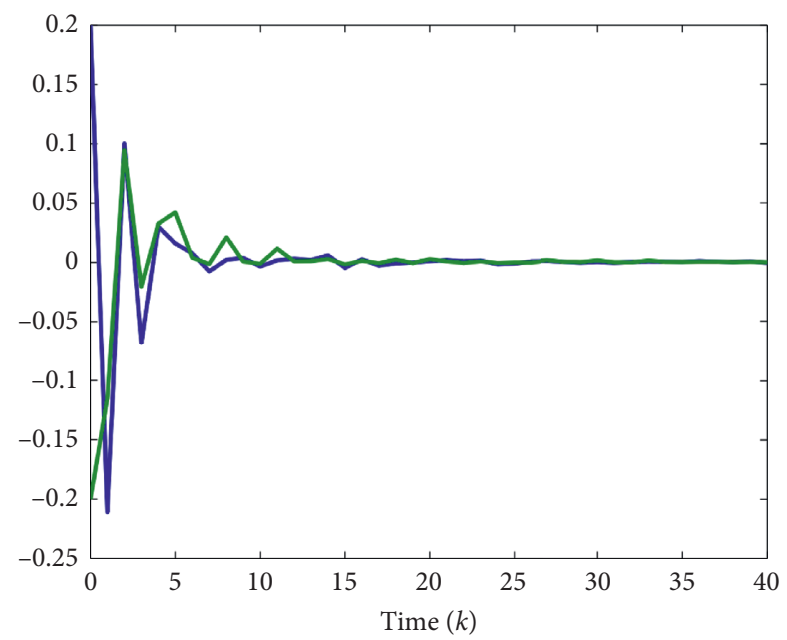

$-\delta_{1}(k)$
$-\delta_{2}(k)$

FIgUre 3: The state trajectories with CF.

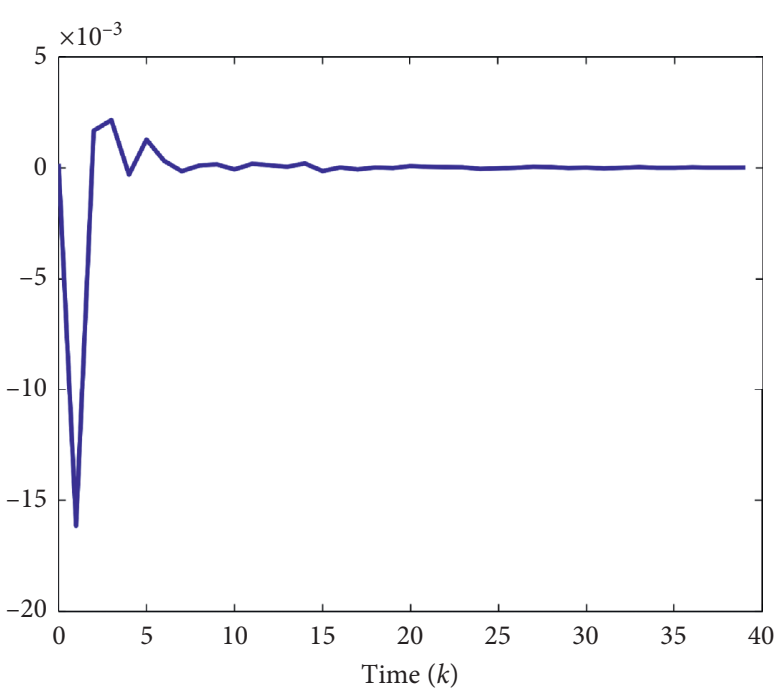

$-u(k)$

Figure 4: Control input.

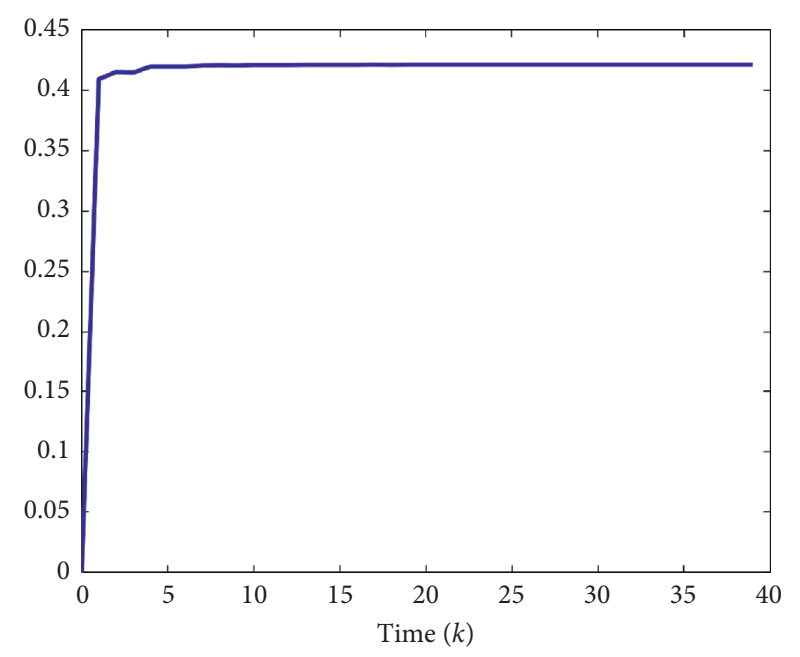

$-\gamma_{d}(k)$

Figure 5: Evolution of $\gamma^{d(k)}$.

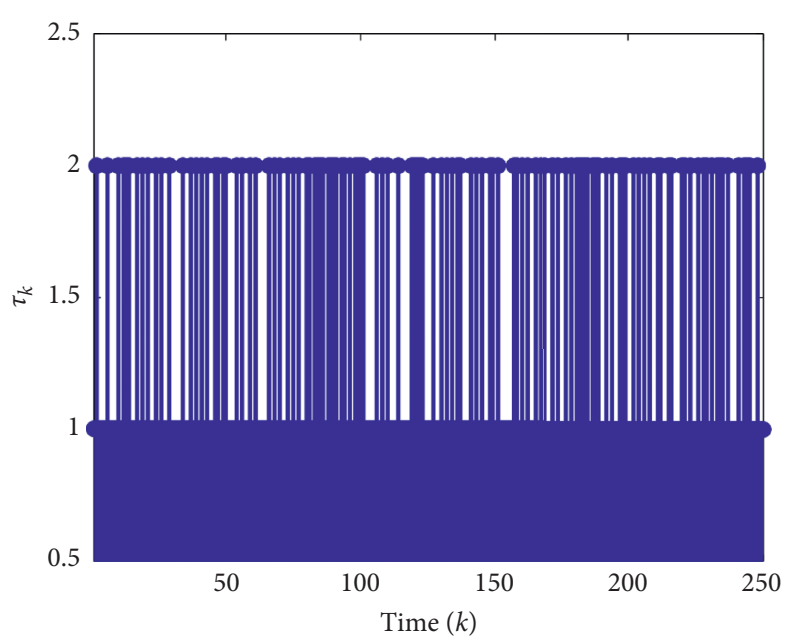

Figure 6: State mode. 


$$
\begin{aligned}
A_{1 \mu} & =\left[\begin{array}{cc}
1 & \mathscr{T} \\
\frac{\mathfrak{T} g \mathscr{L}_{\mu}}{\mathscr{J}_{\mu}} & 1-\frac{\mathscr{T} \mathscr{R}}{\mathscr{J}_{\mu}}
\end{array}\right], \\
B_{1 \mu} & =\left[\begin{array}{c}
0 \\
\mathscr{T} \\
\mathscr{J}_{\mu}
\end{array}\right], \\
C_{1 \mu} & =\left[\begin{array}{c}
0 \\
\mathscr{T}
\end{array}\right], D_{1 \mu}=[10], F_{1 \mu}=1, \\
A_{2 \mu} & =\left[\begin{array}{c}
\mathscr{T} \\
\mathcal{T} g \mathscr{L}_{\mu} \\
\mathcal{E} \frac{\mathscr{T}}{\mathscr{J}_{\mu}} 1-\frac{\mathscr{T} \mathscr{R}}{\mathscr{J}_{\mu}}
\end{array}\right], \\
B_{2 \mu} & =\left[\begin{array}{l}
0 \\
\mathscr{T} \\
\mathscr{J}_{\mu}
\end{array}\right], \\
C_{2 \mu} & =\left[\begin{array}{l}
0 \\
\mathscr{T}
\end{array}\right],
\end{aligned}
$$

The TPM $\Gamma$ and CPM $\Lambda$ are expressed as follows:

$$
\begin{aligned}
\Gamma & =\left[\begin{array}{cc}
0.4 & 0.6 \\
0.75 & 0.25
\end{array}\right], \\
\Lambda & =\left[\begin{array}{cc}
0.7 & 0.3 \\
0.45 & 0.55
\end{array}\right] .
\end{aligned}
$$

Assuming that $L=1$ and $\alpha_{0}=0.81, \alpha_{1}=0.2236$, $\bar{\alpha}_{0}=0.13$, and $\bar{\alpha}_{1}=0.2236$. Meanwhile, $\omega(k)$ and $\zeta(k)$ are chosen as Example 4.1. Solving the inequalities of Theorem 2 , the controller gains are achieved:

$$
\left[\begin{array}{ll}
K_{11} & K_{12} \\
K_{21} & K_{22}
\end{array}\right]=\left[\begin{array}{cccc}
2.4702 & -10.3037 & 2.5196 & -9.9710 \\
-0.4982 & -8.7620 & -0.4263 & -7.9807
\end{array}\right] \text {. }
$$

For initial condition $\delta(0)=[0.2-0.3]^{\top}$, by resorting to the obtained filter gains, Figures 6 and 7 demonstrate the state mode and controller mode, respectively. The state trajectories with CF are shown in Figure 8, and control input is depicted in Figure 9. In the end, Figure 10 plots the evolution of $\gamma_{d}(k)$. It can be observed from Figures $8-10$ that the proposed controller is effective.

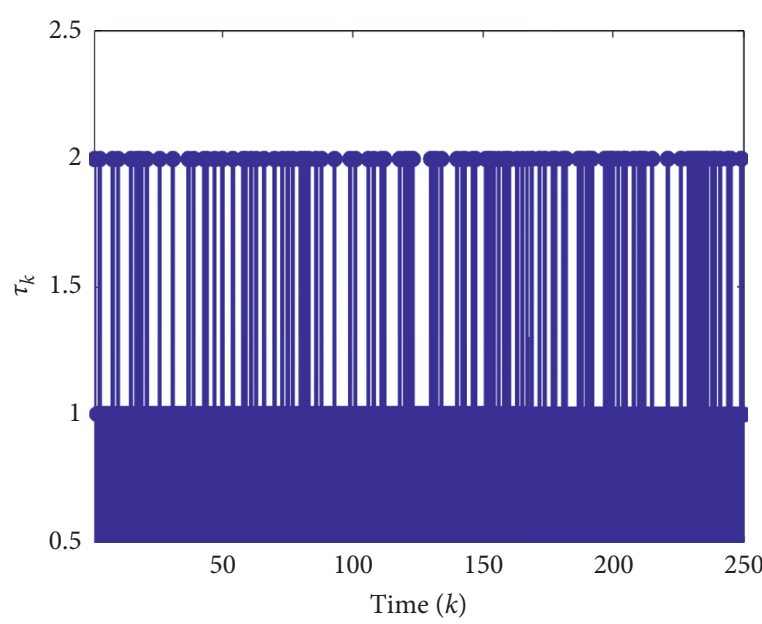

FIGURE 7: Controller mode.

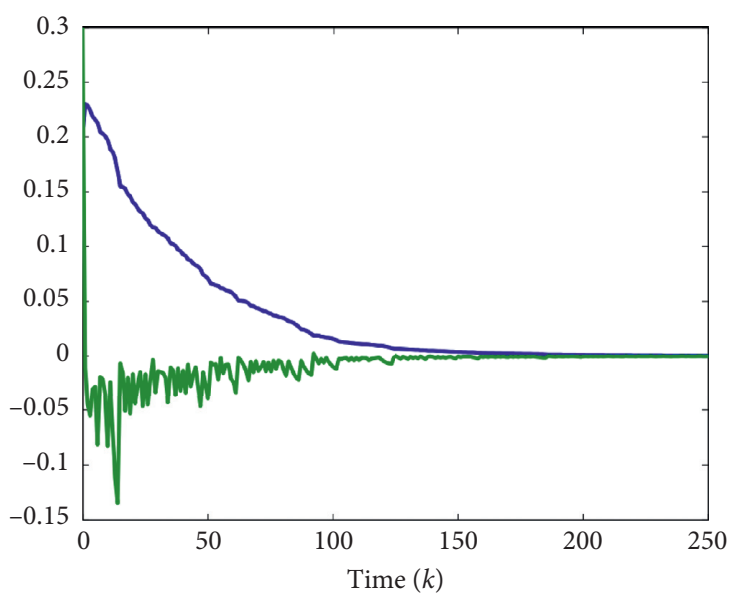

$\delta_{1}(k)$

$\delta_{2}(k)$

Figure 8: The state trajectories with CF.

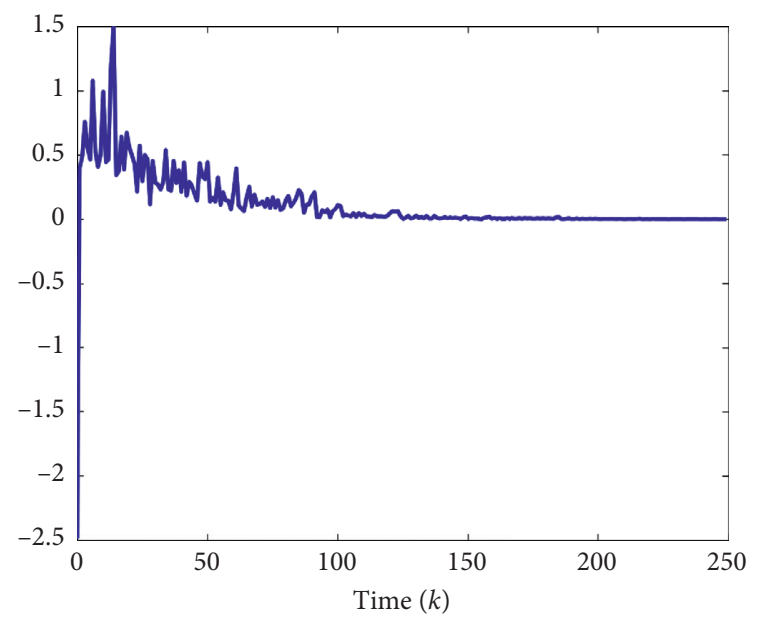

$-u(k)$

Figure 9: Control input. 


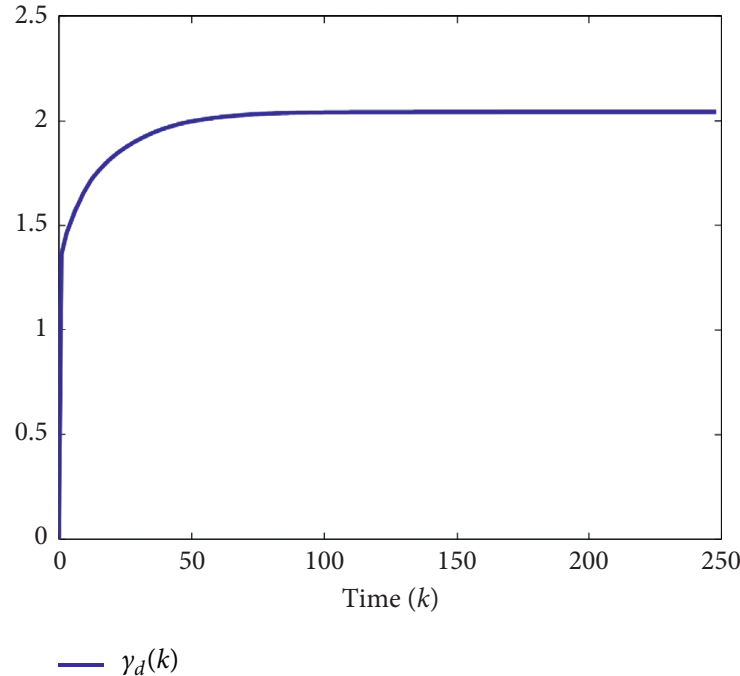

Figure 10: Evolution of $\gamma^{d(k)}$.

\section{Conclusions}

In this work, the asynchronous control for FMSSs with randomly occurring FC is designed. By resorting to the T-S fuzzy model, the nonlinear MSSs can be handled. Furthermore, the Rice fading model is proposed to capture the randomly occurring channel fading, which covers packet dropouts and network-induced delays as special cases. In light of HMM, the asynchronous phenomenon of controller is taken into consideration, and asynchronous fuzzy controller is obtained. In the end, two examples are applied to demonstrate the validity of the derived results. It can be observed that, compared with the existing approach, the obtained methodology is more general, and less conservatism results are expressed. In the networked scenario, sliding mode control for FMSSs with FC is significant in future works [32].

\section{Data Availability}

The data used to support the findings of this study are included within the article.

\section{Conflicts of Interest}

The authors declare that they have no conflicts of interest.

\section{Acknowledgments}

This work was supported by the Science and Technology Project of State Grid Jiangxi Electric Power Co. Ltd. (521820200036).

\section{References}

[1] R. Rajagopalan and P. Varshney, "Connectivity analysis of wireless sensor networks with regular topologies in the presence of channel fading," IEEE Transactions on Wireless Communications, vol. 8, no. 7, pp. 3475-3483, 2009.
[2] L. Su and G. Chesi, "Robust stability analysis and synthesis for uncertain discrete-time networked control systems over fading channels," IEEE Transactions on Automatic Control, vol. 62, no. 4, pp. 1966-1971, 2017.

[3] N. Elia, "Remote stabilization over fading channels," Systems \& Control Letters, vol. 54, no. 3, pp. 237-249, 2005.

[4] J. Song, Y. Niu, and S. Wang, "Robust finite-time dissipative control subject to randomly occurring uncertainties and stochastic fading measurements," Journal of the Franklin Institute, vol. 354, no. 9, pp. 3706-3723, 2017.

[5] J. Li and Y. Niu, "Sliding mode control subject to rice channel fading," IET Control Theory \& Applications, vol. 13, no. 16, pp. 2529-2537, 2019.

[6] S. Zhang, Z. Wang, D. Ding, H. Dong, F. E. Alsaadi, and T. Hayat, "Nonfragile $H_{\infty}$ fuzzy filtering with randomly occurring gain variations and channel fadings," IEEE Transactions on Fuzzy Systems, vol. 24, no. 3, pp. 505-518, 2016.

[7] H. Dong, Z. Wang, S. X. Ding, and H. Gao, "Event-based $H_{\infty}$ filter design for a class of nonlinear time-varying systems with fading channels and multiplicative noises," IEEE Transactions on Signal Processing, vol. 63, no. 13, pp. 3387-3395, 2015.

[8] P. Cheng, J. Wang, S. He, X. Luan, and F. Liu, "Observerbased asynchronous fault detection for conic-type nonlinear jumping systems and its application to separately excited DC motor," IEEE Transactions on Circuits and Systems I: Regular Papers, vol. 67, no. 3, pp. 951-962, 2020.

[9] J. Cheng, J. H. Park, J. Cao, and W. Qi, "Asynchronous partially mode-dependent filtering of network-based MSRSNSs with quantized measurement," IEEE Transactions on Cybernetics, vol. 50, no. 8, pp. 3731-3739, 2020.

[10] J. Cheng, J. H. Park, X. Zhao, H. R. Karimi, and J. Cao, "Quantized nonstationary filtering of network-based Markov switching RSNSs: a multiple hierarchical structure strategy," IEEE Transactions on Automatic Control, p. 1. 2019, In press.

[11] J. Cheng, J. H. Park, J. Cao, and W. Qi, "A hidden mode observation approach to finite-time SOFC of Markovian switching systems with quantization," Nonlinear Dynamics, vol. 100, pp. 509-521, 2020.

[12] J. Cheng, Y. Shan, J. Cao, and J. H. Park, "Nonstationary control for T-S fuzzy Markovian switching systems with variable quantization density," IEEE Transactions on Fuzzy Systems, p. 1. 2020, In press.

[13] H. Shen, F. Li, S. Y. Xu, and V. Sreeram, "Slow state variables feedback stabilization for semi-Markov jump systems with singular perturbations," IEEE Transactions on Automatic Control, vol. 63, no. 8, pp. 2709-2714, 2018.

[14] M. Ali and R. Saravanakumar, "Improved $H_{\infty}$ performance analysis of uncertain Markovian jump systems with overlapping time-varying delays," Complexity, vol. 21, no. S1, pp. 460-477, 2016.

[15] Y. Shen, Z. G. Wu, P. Shi, Z. Shu, and H. R. Karimi, " $H_{\infty}$ Control of Markov jump time-delay systems under asynchronous controller and quantizer," Automatica, vol. 99, pp. 352-360, 2019.

[16] Z. G. Wu, P. Shi, Z. Shu, H. Su, and R. Lu, "Passivity-based asynchronous control for Markov jump systems," IEEE Transactions on Automatic Control, vol. 62, no. 4, pp. 20202025, 2017.

[17] P. Cheng, S. He, J. Cheng, X. Luan, and F. Liu, “Asynchronous output feedback control for a class of conic-type nonlinear hidden Markov jump systems within a finite-time interval," IEEE Transactions on Systems, Man, and Cybernetics: Systems, pp. 1-8, 2020. 
[18] L. Ma, X. Huo, X. Zhao, and G. Zong, "Observer-based adaptive neural tracking control for output-constrained switched MIMO nonstrict-feedback nonlinear systems with unknown dead zone," Nonlinear Dynamics, vol. 99, pp. 1019-1036, 2020.

[19] L. Ma, X. Huo, X. Zhao, and G. Zong, "Adaptive fuzzy tracking control for a class of uncertain switched nonlinear systems with multiple constraints: a small-gain approach," International Journal of Fuzzy Systems, vol. 21, pp. 2609-2624, 2019.

[20] G. M. Zhuang, S. F. Su, J. Xia, and W. Sun, "HMM-based asynchronous $H_{\infty}$ filtering for fuzzy singular Markovian switching systems with retarded time-varying delays," IEEE Transactions on Cybernetics, 2020.

[21] T. Wu, J. Cao, L. Xiong, and H. Zhang, "New stabilization results for semi-Markov chaotic systems with fuzzy sampled-data control," Complexity, vol. 2019, Article ID 7875305, 2019.

[22] Y. Wang, H. R. Karimi, H. K. Lam, and H. Yan, "Fuzzy output tracking control and filtering for nonlinear discrete-time descriptor systems under unreliable communication links," IEEE Transactions on Cybernetics, vol. 50, no. 6, pp. 23692379, 2020.

[23] Y. Chang, Y. Wang, F. E. Alsaadi, and G. Zong, "Adaptive fuzzy output-feedback tracking control for switched stochastic pure-feedback nonlinear systems," International Journal of Adaptive Control and Signal Processing, vol. 33, no. 10, pp. 1567-1582, 2019.

[24] H. Ni, Z. Xu, J. Cheng, and D. Zhang, "Robust stochastic sampled-data-based output consensus of heterogeneous multiagent systems subject to random DoS attack: a Markovian jumping system approach," International Journal of Control, Automation and Systems, vol. 17, no. 7, pp. 1687-1698, 2019.

[25] B. Wang, J. Cheng, and X. Zhou, "A multiple hierarchical structure strategy to quantized control of Markovian switching systems," Applied Mathematics and Computation, vol. 373 , p. $125037,2020$.

[26] J. Cheng, D. Zhang, W. Qi, J. Cao, and K. Shi, "Finite-time stabilization of T-S fuzzy semi-Markov switching systems: a coupling memory sampled-data control approach," Journal of The Franklin Institute, 2019, In Press.

[27] J. Cheng, B. Wang, J. H. Park, and W. Kang, "Sampled-data reliable control for T-S fuzzy semi-Markovian jump system and its application to single-link robot arm mode," IET Control Theory and Applications, vol. 11, no. 12, pp. 1904-1912, 2017.

[28] R. Zhang, X. Liu, D. Zeng, S. Zhong, and K. Shi, "A novel approach to stability and stabilization of fuzzy sampled-data Markovian chaotic systems," Fuzzy Sets and Systems, vol. 344, pp. 108-128, 2018.

[29] K. Shi, J. Wang, S. Zhong, Y. Tang, and J. Cheng, "Non-fragile memory filtering of T-S fuzzy delayed neural networks based on switched fuzzy sampled-data control," Fuzzy Sets and Systems, vol. 394, pp. 40-64, 2020.

[30] K. Shi, J. Wang, Y. Tang, and S. Zhong, "Reliable asynchronous sampled-data filtering of TCS fuzzy uncertain delayed neural networks with stochastic switched topologies," Fuzzy Sets and Systems, vol. 381, pp. 1-25, 2020.

[31] P. Cheng and S. He, "Observer-based finite-time asynchronous control for a class of hidden Markov jumping systems with conic-type non-linearities," IET Control Theory and Applications, vol. 14, no. 2, pp. 244-252, 2020.

[32] W. Qi, G. Zong, and H. R. Karimi, "Observer-based adaptive SMC for nonlinear uncertain singular semi-Markov jump systems with applications to DC motor," IEEE Transactions on Circuits and Systems I: Regular Papers, vol. 65, no. 9, pp. 2951-2960, 2018. 